\title{
Study of congenital nystagmus: optokinetic nystagmus
}

\author{
ROBERT D. YEE, ${ }^{1}$ ROBERT W. BALOH, ${ }^{2}$ AND VICENTE HONRUBIA ${ }^{3}$ \\ From the ${ }^{1}$ Department of Ophthalmology, the ${ }^{2}$ Department of Neurology, and the \\ ${ }^{3}$ Division of Head and Neck Surgery, Department of Surgery, UCLA School of Medicine, \\ Los Angeles, California, USA
}

SUMMARY A severe defect of optokinetic nystagmus (OKN) was found in 46 patients with congenital nystagmus. Abnormal patterns of $\mathrm{OKN}$, such as superimposition of pendular oscillations on the optokinetic slow component and inversion of OKN, were observed. Optokinetic gain (eye movement velocity/drum velocity) was decreased compared to that in normal subjects, and optokinetic after-nystagmus, or transient persistence of OKN after cessation of visual stimulation, was absent. These findings suggest that there is a defect in the subcortical optokinetic system in congenital nystagmus. Optokinetic responses did not clearly differentiate patients who had ocular lesions that impair vision, such as ocular albinism and opacities of the ocular media, from patients who did not have such lesions. Similar abnormal patterns of OKN were found in both groups of patients, although optokinetic gain tended to be lower in patients with ocular lesions than in those without lesions.

Optokinetic nystagmus (OKN) is a jerk nystagmus that is usually induced by movement in a large area of the visual surround during clinical examination of eye movements. Rotation of patterns, such as vertical stripes that occupy most or all of the visual field, produces movement of images across the retina. The slow component of OKN tracks the movement of the pattern and tends to stabilise images on the retina. During naturally occurring head movements the visual surround is stationary, and movement of the eyes produces movement of images on the retina. The vestibulo-ocular response (VOR) that is induced by the head movement produces a slow component in the direction opposite to that of the head movement and, therefore, decreases movement of images on the retina. However, at low frequencies of rotation (less than $1 \mathrm{~Hz}$ ) the VOR alone cannot match the velocity of the head movement and cannot completely stabilise the retinal images. Head movements, by producing movement of images on the retina, also induce an optokinetic response. The slow components of the VOR and optokinetic response are in the same direction and act synergistically to match the velocity of the head movement and more effectively stabilise the retinal images improving vision.

Congenital nystagmus is an ocular motor disorder in which instability of fixation is manifested as

Correspondence to Dr R. D. Yee, Jules Stein Eye Institute, Department of Ophthalmology, UCLA School of Medicine, Los Angeles, California 90024, USA. involuntary oscillations with pendular and complex wave forms. ${ }^{12}$ Its aetiology is essentially unknown. A clinically useful classification of congenital nystagmus based on its pathophysiology is not available. Previous attempts to construct a classification have divided patients into those who had associated ocular lesions, such as ocular albinism and opacities of the ocular media, and those who did not have such lesions. It was proposed that poor vision produced by ocular lesions in the first group led secondarily to instability of fixation, whereas in the second group there was a primary maldevelopment of the ocular motor system. However, there is no difference in nystagmus waveforms between these 2 groups of patients to substantiate this classification. ${ }^{2}$ Careful clinical examination is the most effective means of identifying associated ocular lesions. In this study we attempt to determine if $\mathrm{OKN}$ differs between patients with associated ocular lesions and those without such lesions. Poorly formed, absent, and inverted OKN have been reported in congenital nystagmus. ${ }^{3-7}$ However, study of these responses with eye movement recordings has not been reported in a large number of patients.

\section{Patients and methods}

PATIENTS

Forty-six patients with congenital nystagmus were studied. Each patient received a neuro-ophthalmic examination to measure visual functions, such as 
visual acuity in the position of gaze with least nystagmus (null position), and to detect associated ocular lesions that could impair vision, such as ocular albinism, chorioretinal scars, and optic atrophy. Twenty-eight patients had no other associated ocular lesion that could impair vision and were classified as having motor-defect nystagmus, as described by Cogan. ${ }^{4}$ Visual acuity ranged from $20 / 25$ to 20/60 in this group of patients (average 20/40). Eighteen patients with ocular lesions were classified as having sensory-defect nystagmus. Their visual acuity ranged from 20/40 to 20/400 (average 20/100).

\section{EYE MOVEMENT RECORDINGS}

Horizontal eye movements were recorded monocularly with a D-C electro-oculographic system that had a band width of $0-35 \mathrm{~Hz}(-3 \mathrm{~dB})$. Skin electrodes were placed at the inner and outer canthi of each eye. Electrodes were also placed above and below the left eye to detect artefacts from eyelid blinks. Patients were seated within a 2-metre diameter optokinetic drum whose interior consisted of $2.5-\mathrm{cm}$ wide, white, vertical stripes that were placed every 15 degrees against a black background. Patients were instructed to fixate stripes as they passed in front of them and not to track individual stripes as they traversed their visual fields. The drum was rotated at constant velocities of $10^{\circ}, 30^{\circ}$, and $60 \%$ second toward the right and left, and sinusoidally at $0.05 \mathrm{~Hz}$ and peak velocities of $30^{\circ}$ and $60^{\circ} /$ second. The mean slow component velocity was calculated for approximately 60 seconds (80 to 120 nystagmus cycles) by a laboratory computer after digitisation at 200 samples/second. Eye movements were also recorded during attempted fixation of a small spot in the primary position and in eccentric, horizontal positions at every 5 degrees to 30 degrees. The effects of eccentric gaze on nystagmus characteristics, such as waveform and slow component velocity, were compared to eye movements induced by optokinetic stimulation to verify that the OKN recorded was not simply the result of eccentric gaze.

The gain of the optokinetic response (slow component eye velocity/drum velocity) was then calculated. There is difficulty in attempting to measure OKN quantitatively in congenital nystagmus. The calculation of gain assumes that the movement of the drum is an accurate representation of the movement of images on the retina which is the stimulus for OKN. In congenital nystagmus, however, the spontaneous nystagmus also produces movement of images on the retina. Fortunately the movement of the drum was in one direction and was of constant velocity, while the smooth movements of the spontaneous nystagmus were usually in both directions and had varying velocity, making detection of an effect of optokinetic stimulation easier. An experimental paradigm could be constructed in which retinal images are stabilised during spontaneous nystagmus by a negative feed-back system that detected the nystagmus and moved the drum to match the eye movements. A constant-velocity drum movement could be added, and an 'open-loop' gain of OKN could be measured. However, in the present study without retinal stabilisation the calculated 'gain' was probably a useful approximation of the function of the optokinetic system and was a closer approximation to more commonly used clinical tests of OKN.

The optokinetic response in normal subjects is characterised by a persistence of nystagmus in the dark for several seconds after optokinetic stimulation has been stopped (optokinetic after-nystagmus), and by an illusion of self-rotation in the opposite direction to drum rotation during constant velocity optokinetic stimulation (circularvection). ${ }^{8-10}$ An attempt was made to induce optokinetic afternystagmus (OKAN) in 20 patients with motordefect nystagmus and in 12 patients with sensorydefect nystagmus. After $60 \mathrm{~s}$ of drum rotation at a constant velocity of $60 \%$ s optokinetic stimulation was terminated by turning off the lights and a transient persistence of $\mathrm{OKN}$ was sought. Sixteen patients with motor-defect nystagmus and 10 patients with sensory-defect nystagmus were questioned about circularvection. During 1 minute of constant velocity drum rotation at $60^{\circ} / \mathrm{s}$ patients were asked if they felt that they were moving, and, if so, in what direction they were rotating. After cessation of optokinetic stimulation in the dark they were asked if the illusion of self-rotation persisted (post-circularvection).

\section{Results}

\section{OKN PATTERNS}

\section{Several patterns of OKN were observed.}

Normal. The normal pattern of OKN was a jerk nystagmus with the slow component in the direction of drum rotation and the fast component in the opposite direction (Fig. 1). The velocity of the slow component was constant during the nystagmus cycle, resulting in a ramp-like shape of the slow component. Eleven patients with motor-defect nystagmus $(39 \%)$ and 7 patients with sensory-defect nystagmus $(39 \%)$ displayed this normal OKN pattern during stimulation at the several drum velocities. During optokinetic stimulation the eyes of normal subjects and most patients with congenital nystagmus deviate in the orbit in the direction of 


\section{A}

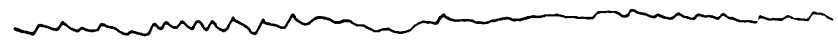

$A$

Fig. 1 Normal pattern of OKN. A. Nystagmus during attempted fixation of a stationary target. Top-10 $0^{\circ}$ to right. Centre-primary position. Bottom $-10^{\circ}$ to left. Note null position at $10^{\circ}$ to left. B. Drum rotating at $60^{\circ} / \mathrm{s}$ to right. Note ramp-like shape of slow components. Arrow indicates centre position. C. Drum rotating at $60^{\circ} / \mathrm{s}$ to left. Arrow indicates centre position. Deflections up are to the right, deflections down are to the left.

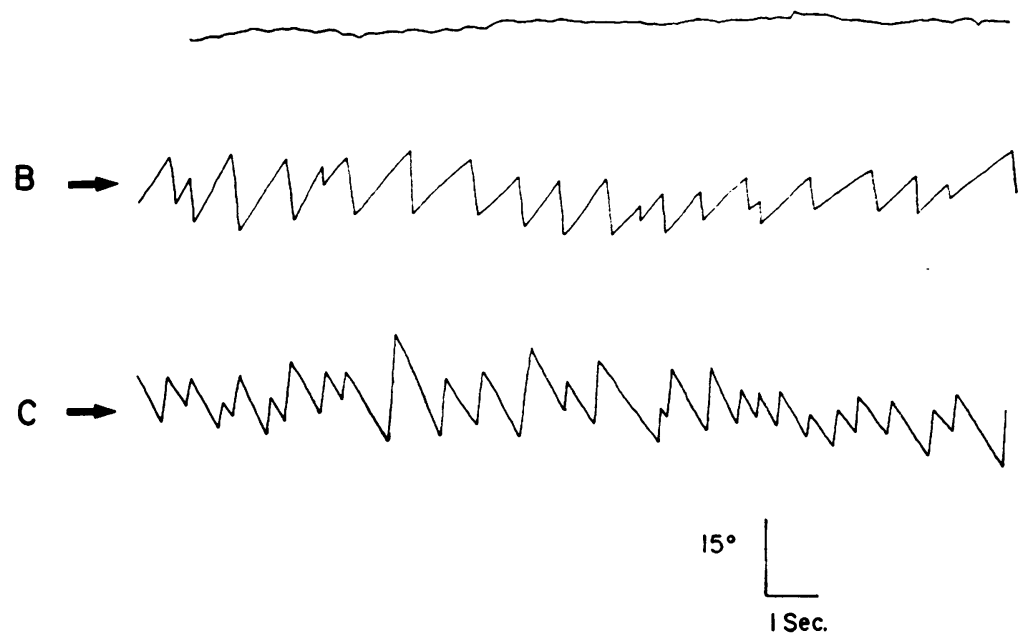

the fast component of $\mathrm{OKN}$, as shown in Fig. 1. The eyes usually do not deviate more than 15 degrees from the midline. The normal pattern of OKN in patients with congenital nystagmus was not produced by eccentric gaze, as could be seen by comparing nystagmus in eccentric gaze to $\mathrm{OKN}$. In Fig. 1 the null position, or position in which the nystagmus was least in amplitude, was approximately 10 degrees to the left. In most patients the nystagmus has a pendular waveform at the null position and a jerk-like waveform in eccentric gaze to either side of the null position. The fast component or saccade in eccentric gaze is usually in the direction of eccentric gaze. The relatively large amplitude, ramp-like slow component of the normal pattern of OKN cannot be explained simply as a result of eccentric gaze.

Superimposition. The superimposition pattern of OKN consisted of a high frequency pendular oscillation superimposed on the ramp-like slow component. The slow component was in the direction of drum rotation. A large amplitude fast component was present in the opposite direction (Fig. 2). In several patients with this OKN pattern the high
Fig. 2 Superimposition pattern of $O K N$. A. Nystagmus during attempted fixation of a stationary target. Top $-15^{\circ}$ to right. Centre-primary position. Bottom-10 $10^{\circ}$ to left. Note small amplitude, $5 \mathrm{~Hz}$ pendular oscillations. B. Drum rotating at $60^{\circ} / \mathrm{s}$ to right. Note ramp-like slow component of $O K N$ during intervals in which pendular oscillations are absent. Arrow indicates centre position. C. Drum rotating at $60^{\circ} / \mathrm{s}$ to left. Arrow indicates centre position. Deflections up are to the right, deflections down are to the left. 


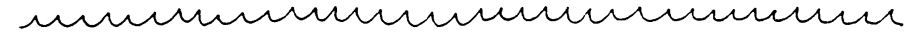

$$
\begin{aligned}
& \text { A }
\end{aligned}
$$

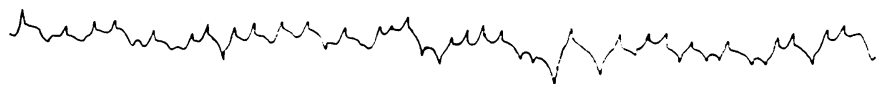

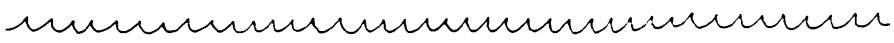

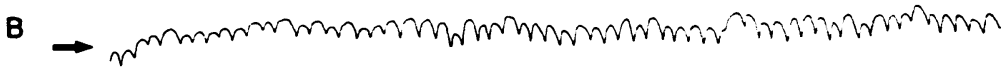

$$
\begin{aligned}
& \text { C }
\end{aligned}
$$

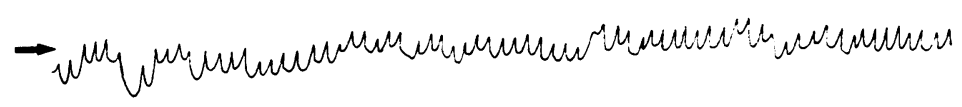

$$
\begin{aligned}
& \left.15^{\circ}\right|_{1 \mathrm{Sec} .}
\end{aligned}
$$

frequency oscillation was absent during part of the slow component ramp, as illustrated in Fig. 2, showing that the pendular oscillation was in fact engrafted on a smooth optokinetic response. The velocity of the slow component ramp was measured during these intervals of absent pendular oscillation. Four patients with motor-defect nystagmus (14\%) and 3 patients with sensory-defect nystagmus (17\%) had this OKN pattern.

Pseudoinversion and true inversion. The pseudoinverted pattern of $\mathrm{OKN}$ consisted of slow components in the direction opposite to drum rotation and fast components in the same direction as drum rotation. This pattern simply represented persistence of the patient's basic nystagmus, that is, the nystagmus pattern during attempted fixation of a stationary target, during optokinetic stimulation rather than true inversion of the optokinetic response. For example, if the saccade in the basic nystagmus waveform was towards the left, during drum rotation to the right there might be little effect on the basic nystagmus, but the saccade towards the left would give the impression that the optokinetic response was in the expected direction. During drum rotation to the left the basic nystagmus would persist, but the saccade toward the left would give the impression that the OKN was inverted. The clinically observed 'inversion' would be present in only 1 direction of drum rotation. Two patients with sensory-defect nystagmus $(22 \%)$ had this pattern. No patient with motor-defect nystagmus had this pattern.

Some patients showed pseudoinversion of OKN in both directions of drum rotation. In this pattern the saccade of the basic nystagmus waveform was used to track stripe movement at the lower drum velocities of 10 and 30 degrees/s. In Fig. 3 the basic nystagmus consisted of alternating nystagmus cycles with small saccades to the right and left. Dell'Osso and Daroff ${ }^{1}$ have shown that the saccade in each cycle attempts to refoveate the target. During drum rotation to the right the nystagmus cycle with the saccade to the right predominated. During rotation to the left the cycle with the saccade to the left predominated. However, close examination of the recorded eye movements showed that OKN was not actually inverted. The smooth eye movements of the nystagmus pattern during drum rotation in both directions had exponentially increasing velocities rather than the constant velocities of the ramp-like slow components of the normal OKN pattern. These smooth movements represent the smooth movements of the basic nystagmus waveform and not inverted slow components of OKN. As shown in Fig. 3, this pattern of 'pseudoinversion' of OKN was not produced by the effect of eccentric gaze on the basic nystagmus. Three patients with motor-defect nystagmus (11\%) and 2 patients with sensory-defect nystagmus $(11 \%)$ had this pattern.

True inversion of $\mathrm{OKN}$ with reversal of ramp-like slow components was found in only 2 patients with motor-defect nystagmus $(7 \%)$ and in 2 patients with sensory-defect nystagmus $(11 \%), 1$ patient with ocular albinism, and 1 patient with oculocutaneous albinism. In Fig. 4 the slow components of OKN were ramp-like with constant velocity. A gradual build-up of reversal during constant velocity drum rotation was occasionally observed. Therefore most instances of 'inversion' of OKN detected by clinical 
Fig. 4 Inverted pattern of OKN. A. Nystagmus during attempted fixation of a stationary target. Top- $15^{\circ}$ to right. Centre-primary position. Bottom- $15^{\circ}$ to left. Note null position at $15^{\circ}$ to left. B. Drum rotating at $30^{\circ} / \mathrm{s}$ to right. Note slow components are ramp-like and to the left. Arrow indicates centre position. C. Drum rotating at $30 \%$ s to left. Note slow components are to the right. There is gradual build-up of slow component velocity. Arrow indicates centre position. Deflections up are to the right, deflections down are to the left.
A

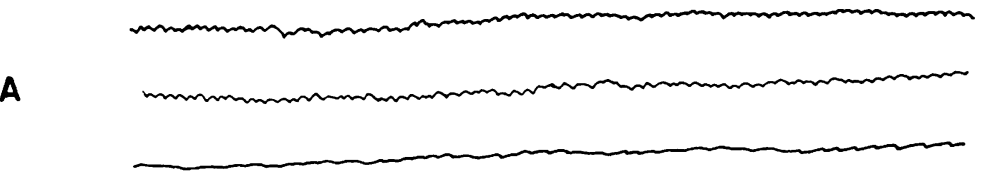

B
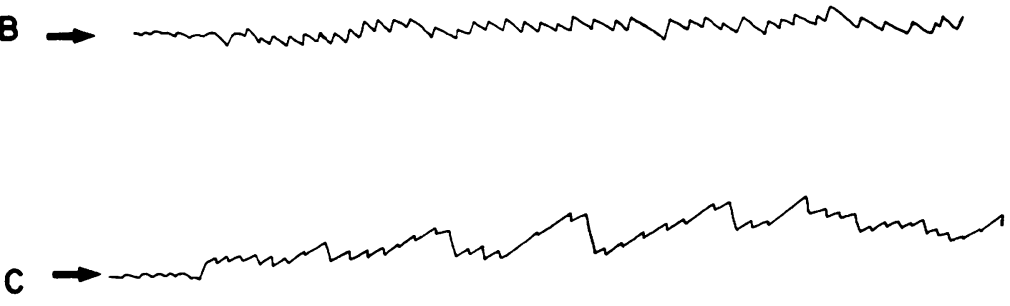

15. $\bigsqcup_{\text {ISec. }}$ observation alone were found to represent pseudoinversion rather than true inversion when examined by eye movement recordings.

\section{OPTOKINETIC GAIN}

Gain was not measured in patients with the pseudoinverted or true inverted pattern of OKN. Twentythree patients with motor-defect nystagmus $(82 \%)$ had optokinetic responses in which the OKN gain could be measured; whereas only 10 patients with sensory-defect nystagmus $(56 \%)$ had measurable OKN gains. The gains from constant velocity tests and sinusoidal tests are presented in Table 1. The data from constant velocity drum rotation to the right are presented. Data from leftward drum rotation were similar. The OKN gain in motordefect nystagmus was greater than that in sensorydefect nystagmus. For example, during constant velocity drum rotation at $30^{\circ} / \mathrm{s}$ the mean OKN gain \pm 1 SD for patients with motor-defect nystagmus was $0.38 \pm 0 \cdot 19$, whereas the gain in patients with sensory-defect nystagmus was $0 \cdot 22 \pm 0 \cdot 19$. The mean OKN gains for both groups were significantly different from that of normal subjects in our laboratory, $0 \cdot 81 \pm 0.09 . .^{11}$ The gain in patients with motordefect nystagmus was significantly greater than that in patients with sensory-defect nystagmus $(P<0.01$, Student's $t$ test). The difference between the patient groups at $60^{\circ} / \mathrm{s}$ was significant at the $1 \%$ level, but the difference at $10 \%$ was not significant at the $5 \%$ level. During sinusoidal drum rotation $(0.05 \mathrm{~Hz}$, $30 \%$ ), the mean gains in both patient groups were significantly lower than in normal subjects $(\mathbf{P}<0.01$, Student's $t$ test), and were significantly different from each other $(P<0.02$, Student's $t$ test $)$. The mean gain in motor-defect nystagmus was $0.39 \pm$ $0 \cdot 14$, and in sensory-defect nystagmus was $0.16 \pm$ $0 \cdot 18$. The mean gain in normal subjects in our laboratory was $0 \cdot 75 \pm 0 \cdot 15$. The difference between the patient groups at $60 \%$ was not significant at the $5 \%$ level.

The hypothesis that OKN gain was linearly related to visual acuity was evaluated in all patients and within each patient group. However, linear

Table 1 Optokinetic gain 1

\begin{tabular}{|c|c|c|c|c|c|}
\hline & \multicolumn{3}{|c|}{ Constant drum velocity ${ }^{2}$} & \multicolumn{2}{|c|}{ Peak sinusoidal drum velocity } \\
\hline & $10^{\circ} / \mathrm{s}$ & $30^{\circ} / \mathrm{s}$ & $60^{\circ} / \mathrm{s}$ & $30^{\circ} / \mathrm{s}$ & $60^{\circ} / \mathrm{s}$ \\
\hline Normal subjects & $0.88 \pm 0.07^{4}$ & $0.81 \pm 0.09$ & $0.79 \pm 0.13$ & $0.75 \pm 0.15$ & $0.71 \pm 0.22$ \\
\hline Motor-defect nystagmus & $0 \cdot 29 \pm 0 \cdot 13$ & $0 \cdot 38 \pm 0 \cdot 19$ & $0 \cdot 35 \pm 0 \cdot 18$ & $0 \cdot 39 \pm 0 \cdot 14$ & $0 \cdot 25 \pm 0 \cdot 17$ \\
\hline Sensory-defect nystagmus & $0 \cdot 20 \pm 0 \cdot 17$ & $0 \cdot 22 \pm 0 \cdot 19$ & $0 \cdot 19 \pm 0 \cdot 19$ & $0 \cdot 16 \pm 0 \cdot 18$ & $0 \cdot 18 \pm 0 \cdot 12$ \\
\hline
\end{tabular}

${ }^{1} \mathrm{Gain}=$ peak eye velocity $/$ peak drum velocity. ${ }^{2}$ Constant drum rotation to the right. ${ }^{3}$ Sinusoidal drum rotation at $0.05 \mathrm{~Hz} .{ }^{4} \mathrm{Mean}$ gain $\pm 1 \mathrm{SD}$. 
regression analysis indicated that correlation coefficients of OKN gain and visual acuity were not statistically significant among all patients (0.577, $P>0.05)$, within the motor-defect group $(0.524$, $P>0.05)$ or within the sensory-defect group $(0.299$, $\mathrm{P}>0.05$ ).

OKAN AND CIRCULARVECTION

None of the 32 patients tested had OKAN after constant velocity optokinetic stimulation in either direction. Some patients had persistence of their basic nystagmus with eyes opened in the dark with or without previous optokinetic stimulation. This nystagmus was not interpreted as representing OKAN. Eighteen patients with motor-defect nystagmus $(64 \%)$ and 7 patients with sensory-defect nystagmus $(40 \%)$ experienced circularvection (CV). Characteristically, the patients reported an illusion of self-rotation in the direction opposite to that of drum rotation beginning 5 to 10 seconds after onset of optokinetic stimulation. CV ended abruptly when stimulation was terminated by turning off the lights. No correlation between OKN gain and $\mathrm{CV}$ was found in either patient group.

\section{Discussion}

Convincing evidence has been presented that $\mathrm{OKN}$ results from simultaneous stimulation of a subcortical optokinetic system and the pursuit system in many species. Ter Braak ${ }^{1213}$ showed that afoveate animals, such as the rabbit, produced $\mathrm{OKN}$ in response to movement of large objects (passive OKN) but did not track small objects of possible 'interest' to the animal. Passive OKN persisted after removal of the cerebral hemispheres in these animals and was presumably produced by an entirely subcortical ocular motor system. Animals with a fovea or area centralis, such as the monkey and $\mathrm{dog}$, had both passive and active OKN. Only active OKN was lost after removal of the cerebral hemispheres or occipital lobes, and presumably utilised cortical pathways.

Recent studies of OKN in human patients with localised lesions affecting the ocular motor system ${ }^{14-16}$ suggest that the optokinetic response in man consists of contributions from the smooth pursuit system and a subcortical optokinetic system. The smooth pursuit contribution depends on stimulation of the fovea, utilises the retino-geniculocalcarine sensory pathway and the occipito-mesencephalic motor pathway, and produces the rapid build-up of slow component velocity of OKN observed after a step of optokinetic stimulation. The contribution from the subcortical system is similar to $\mathrm{OKN}$ in afoveate animals, such as the rabbit. ${ }^{17}$ This contribution depends on stimulation of motion-sensitive cells in the retina ${ }^{18-20}$ that project via the accessory optic tract to cells in the nucleus of the optic tract. ${ }^{21}$ There is convincing evidence that motion-sensitive retinal ganglion cells are the input stage of the optokinetic loop in the rabbit, since their firing rates have been shown to correlate with the velocity of $\mathrm{OKN} .{ }^{22}$ Cells in the nucleus of the optic tract in turn project to the vestibular nuclei and cerebellum. ${ }^{23}$ The optokinetic response in afoveate animals is characterised by a slow, gradual build-up of slow component velocity over many seconds and OKAN. In several patients with localised lesions of the parietal lobe ${ }^{15}$ and cerebellar flocculi ${ }^{16}$ whom we have reported on OKN was characterised by a slow build-up of slow component velocity and preservation of OKAN. Smooth pursuit was severely impaired such that the subcortical contribution to OKN was 'uncovered'. Ter Braak $^{14}$ described a man who was cortically blind from histopathologically verified infarction of the occipital lobes, who had no pursuit, but who showed the persistence of OKN with a slow build-up and OKAN with rotation of a surrounding optokinetic drum in one direction. Presumably the $\mathrm{OKN}$ in this patient was produced by the subcortical optokinetic system.

The pursuit system is probably abnormal in congenital nystagmus. The function of this system is difficult to measure, since the smooth movement velocities of the spontaneous nystagmus are very high (up to $90^{\circ} / \mathrm{s}$ ) and a relatively small velocity contribution from pursuit is difficult to detect. Pursuit function has been measured in a few patients with relatively low velocities and has been shown to be subnormal. ${ }^{24}$

In normal human subjects OKAN (present in approximately $70 \%$ of subjects in our laboratory) circularvection and postcircularvection in the dark probably result from stimulation of the subcortical system. ${ }^{8-10}$ In our patients with congenital nystagmus slow build-up of OKN, OKAN, and post circularvection were not observed. In most patients nystagmus was greatly reduced in amplitude or absent in the dark with loss of fixation. We would expect that OKAN could be easily observed if it were present. Although the slow component of OKN could be obscured by the high velocity smooth movements of the basic nystagmus in the light, the low-velocity slow component of OKAN should be detectable in the dark. The absence of OKAN in our patients was another indication that a normal optokinetic response was not simply hidden by the intense basic nystagmus. Most of our patients did experience circularvection during optokinetic stimulation, however. Circularvection does not appear to 
correlate with $\mathrm{OKN}$ in congenital nystagmus. A severe defect in the subcortical optokinetic system, therefore, appears to be present. It is unlikely that the impaired OKN in congenital nystagmus can be explained simply by poor visual acuity. Defects of central vision resulting from acquired lesions of the macula in man can be associated with normal OKN. ${ }^{25}$

The abnormalities of OKN in patients with motor-defect nystagmus were qualitatively similar to but quantitatively different from those in patients with sensory-defect nystagmus. This suggests that the type and location of defect(s) are similar in both groups but that the severity is variable. In motordefect nystagmus we might hypothesise that developmental anomalies are present in the pursuit and subcortical optokinetic systems at birth. A degree of postnatal development might be required in these systems. In sensory-defect nystagmus the absence of well-formed retinal images or normal conduction through the optic nerves due to bilateral opacities of the ocular media or bilateral optic atrophy could interrupt this postnatal development. Alternatively, in sensory-defect nystagmus 2 distinct, unrelated defects in the visual afferent and ocular motor systems may be present. The ocular motor lesion would be identical to that in motor-defect nystagmus. Although no basic differences have been found between motor-defect nystagmus and sensorydefect nystagmus in nystagmus waveform, frequency, and amplitude, ${ }^{2}$ or in patterns of $\mathrm{OKN}$, this classification of congenital nystagmus is still clinically useful. It emphasises the need to search carefully for associated ocular lesions. The presence of lesions has important implications for the prognosis for useful visual function and evaluating clinical indications for prism and surgical therapies. We have found that measurement of the patient's best visual acuity in the null position and/or with convergence in which the nystagmus amplitude is minimised is helpful in indicating that associated ocular lesions are present. Visual acuity is usually less than $20 / 80$ in sensory-defect nystagmus and better than 20/60 in motor-defect nystagmus despite similar amplitude of nystagmus. Careful clinical ophthalmic examination, including measurement of visual acuity in the null position and tests of colour vision, is still the best means of detecting associated ocular lesions.

This work was supported by a grant from the National Eye Institute, NIH Grant 2 R0I EY01853-03.

\section{References}

1 Dell'Osso LF, Daroff RB. Congenital nystagmus waveforms and foveation strategy. Doc Ophthalmol 1975; 39: $155-82$.

2 Yee RD, Wong EK, Baloh RW, Honrubia V. A study of congenital nystagmus: waveforms. Neurology 1976; 26: 326-33.

3 Brunner $H$. Über Inversion des experimentellen optokinetischn Nystagmus. Z Ohrenheilkunde 1921; 55: 574.

4 Cogan DG. Congenital nystagmus. Can J Ophthalmol 1967: 2: 4-10.

5 Smith JL. Nystagmus. Am Orthopt J 1973; 23: 27-9.

6 Dichgans J, June R. Oculomotor abnormalities due to cerebellar lesions. In Lennerstrand G, Bach-y-Rita P, eds. Basic Mechanisms of Ocular Motility and their Clinical Implications. Oxford: Pergamon Press, 1975: 288.

7 Hood JD. Leech J. The significance of peripheral vision in the perception of movement. Acta Otolaryngol (Stockh) 1974; 77: 72-9.

8 Cohen B, Matsuo V, Raphan T. Quantitative analysis of the velocity characteristics of optokinetic nystagmus and optokinetic after nystagmus. $J$ Physiol $1977 ; 270$ : 321-44.

9 Cohen B, Uemura T, Takemori S. The effects of labyrinthectomy on optokinetic nystagmus (OKN) and optokinetic after-nystagmus (OKAN). Equil Res 1973; 3: 88-93.

10 Brandt T, Dicans J, Büchele W. Motion habituation: inverted self-motion perception and optokinetic after nystagmus. Exp Brain Res 1974; 21 : 337-52.

11 Yee RD, Jenkins HA, Baloh RW, Honrubia V, Lau CGY. Vestibular-optokinetic interactions in normal subjects and in patients with peripheral vestibular dysfunction. J Otolaryngol 1978; 7: 310-9.

12 Ter Braak JWG. Untersuchen über optokinetischen nystagmus. Arch Neerlandaises Physiol 1936; 21 : 309-76.

13 Ter Braak JWG, Van Vliet AGM. Subcortical optokinetic nystagmus in the monkey. Psychiatr Neurol Neurochir (Amst) 1963; 66: 277-83.

14 Ter Braak JWG, Schenk VWD. Visual reactions in a case of long-standing cortical blindness. J Neurol Neurosurg Psychiatry 1971: 34: 140-7.

15 Baloh RW, Yee RD, Honrubia V. Optokinetic nystagmus in parietal lobe lesions. Ann Neurol 1980; 7: 269-76.

16 Yee RD, Baloh RW, Honrubia V, Lau CGY, Jenkins HA. Slow build-up of optokinetic nystagmus associated with downbeat nystagmus. Invest Ophthalmol Visual Sci 1979; 18: 622-9.

17 Collewijn H. Optokinetic eye movements in the rabbit. Vision Res 1969; 9: 117-32.

18 Rodieck RW. Visual pathways. Annu Rev Neurosci 1979; 2: 193-225.

19 Oyster CW, Barlow HB. Direction-selective units in the rabbit retina: distribution of preferred directions. Science 1967; 155: 841-2.

20 Dowling JE. The vertebrate retina. In: Tower DB, ed. The Nervous System. New York: Raven, 1975; 1: The Basic Neurosciences: 91-100.

21 Collewijn H. Direction-selective units in the rabbit's nucleus of the optic tract. Brain Res 1975; 100: 489-508.

22 Collewijn H, Oyster CW, Takahashi E. Rabbit optokinetic reactions and retinal direction-selective cells. Bibl Ophthalmol 1972; 82: 280-7.

23 Simpson JL, Alley KE. Visual climbing fiber input to rabbit vestibulo-cerebellum: a source of directionspecific information. Brain Res 1974; 82: 302-8.

24 Yamazaki, A. Abnormalities of smooth pursuit and vestibular eye movements in congenital jerk nystagmus. In: Shimizu K, Oosterhuis JA, eds. Proceedings of the XXIII International Congress of Ophthalmology. 1978, 2: $1162-5$.

25 Hood JD. Observations upon the neurological mechanism of optokinetic nystagmus with especial reference to the contribution of peripheral vision. Acta Otolaryngol (Stockh) 1967; 63: 208-15. 